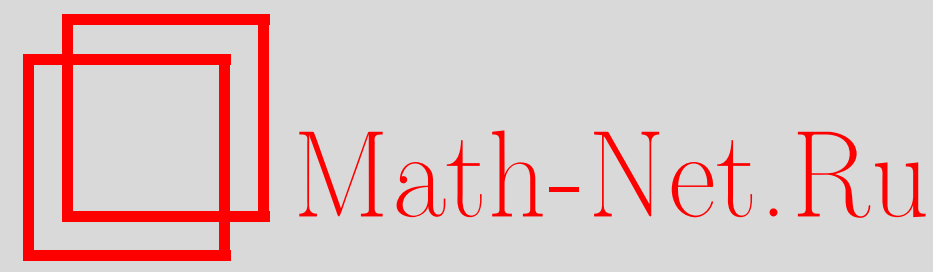

Н. В. Денисова, В. В. Козлов, Полиномиальные интегралы обратимых механических систем с конфигурационным пространством в виде двумерного тора, Матем. сб., 2000, том 191, номер 2, 43-63

DOI: https://doi.org/10.4213/sm452

Использование Общероссийского математического портала Math-Net.Ru подразумевает, что вы прочитали и согласны с пользовательским соглашением http://www . mathnet.ru/rus/agreement

Параметры загрузки:

IP: 3.81 .55 .215

26 апреля 2023 г., 12:37:49 
УДК $517.9+531.01$

\author{
Н. В. Денисова, В.В. Козлов
}

\title{
Полиномиальные интегралы обратимых механических систем с конфигурационным пространством в виде двумерного тора
}

\begin{abstract}
В работе рассматривается задача об условиях существования полиномиальных по импулсам интегралов обратимых гамильтоновых систем. Кинетическая энергия - риманова метрика нулевой кривизны, потенциал - гладкая функция на двумерном торе. Как известно, существование интегралов степени 1 и 2 связано с наличием циклических координат и разделением переменных. Известна также следующая гипотеза: если имеется интеграл степени $n$, независимый от интеграла энергии, то обязательно найдется дополнительный интеграл степени 1 или 2. В настоящей работе эта гипотеза доказана для $n=3$ (обобщение теоремы М. Л. Бялого), а для $n=4,5$ или 6 это установлено при некоторых дополнительных предположениях о спектре потенциала.

Библиографоия: 14 названий.
\end{abstract}

\section{§1. Введение}

В работе рассматриваются натуральные механические системы с двумя степенями свободы, конфигурационным пространством которых является двумерньй тор, допускающие дополнительный полиномиальный первый интеграл по импульсам. Такие системы, очевидно, вполне интегрируемые. Полиномиальные интегралы представимы в виде суммы однородных многочленов по импульсам с гладкими и однозначными коэффициентами на конфигурационном пространстве.

В работе [1] Биркгоф исследовал локальную задачу о наличии линейных и квадратичных интегралов, являющихся полиномами по скоростям. Оказалось, что наличие условного линейного интеграла связано с существованием “скрытой” циклической координаты, а наличие условного квадратичного интеграла позволяет разделить канонические переменные. В работах [2], [3] приведены глобальные варианты этих утверждений для случая, когда конфигурационньм пространством системы является двумерньй тор. Задача о полиномиальных интегралах степени $\leqslant 2$ рассматривалась также в работах [4], [5].

В работе [6] изучена задача о полиномиальных интегралах геодезических потоков на двумерном торе для метрики, у которой конформньй множитель является тригонометрическим многочленом. Установлено, что если геодезический поток

Работа выполнена при финансовой поддержке Российского фонда фундаментальных исследований (грант № 99-01-01096), Федеральной целевой программы "Государственная поддержка интеграции высшего образования и фундаментальной науки" (проект № 2.1-294), а также программы "Университеты России" (проект № 5581).

$$
\text { (C) Н. В. ДенисовА, В. В. Козлов }
$$


допускает дополнительный неприводимый интеграл, полиномиальньй по импульсам, то его степень не превосходит двух. Этот результат был уточнен в [3].

В [7] рассматривалась задача о наличии полного набора независимых полиномиальных интегралов системы с торическим пространством положений $\mathbb{T}^{m}=$ $\left\{x_{1}, \ldots, x_{m} \bmod 2 \pi\right\}$, кинетической энергией

$$
T=\frac{1}{2} \sum_{i, j}^{m} a_{i j} \dot{x}_{i} \dot{x}_{j}, \quad a_{i j}=\text { const }
$$

и потенциалом $V: \mathbb{T}^{m} \rightarrow \mathbb{R}$ в виде тригонометрического многочлена. Оказалось, что полньй набор полиномиальных интегралов существует тогда и только тогда, когда спектр тригонометрического многочлена $V$ лежит на $k \leqslant m$ прямых, попарно ортогонально пересекающихся в начале координат. Показано, что тогда обязательно существуют $m$ независимых интегралов, степени которых $\leqslant 2$. По теореме Вейерштрасса тригонометрические многочлены всюду плотны в пространстве всех гладких функций на торе. Однако метод работы [7] не применим к системам с потенциалом обшего вида.

В [8] отмечено, что натуральная механическая система на двумерном торе с кинетической энергией

$$
T=\frac{1}{2}\left(\dot{x}_{1}^{2}+\dot{x}_{2}^{2}\right)
$$

не может иметь неприводимых интегралов третьей и четвертой степени: в этом случае обязательно имеются интегралы первой и второй степени соответственно. Этот результат получил известность и часто цитируется (см., например, [9]-[11], где обсуждается этот круг вопросов). Однако в [8] доказательство приведено лишь для интеграла третьей степени. К тому же оно содержит пробел, к счастью, не столь существенный для метрики вида (1.2). Однако для метрики общего вида (1.1) заполнение этого пробела требует дополнительных усилий (подробнее об этом - в $\S 4)$. Для интегралов четвертой степени метод работы [8] не проходит уже в самом простом случае с метрикой (1.2).

Полиномиальные интегралы для систем взаимодействующих частиц, когда потенциал имеет вид

$$
\sum_{i<j} f\left(x_{i}-x_{j}\right)
$$

изучались в работе [12]. Во всех найденных интегрируемых случаях периодическая функция $f$ обязательно имеет полюсына вещественной оси. В работе [13] показано, что для периодических потенциалов парного взаимодействия без сингулярностей система с потенциальной энергией (1.3) не может быть вполне интегрируемой.

В настоящей работе рассматривается задача о полиномиальных интегралах $n$-й степени системы с кинетической энергией обшего вида (1.1) и произвольньм аналитическим потенциалом. Получено обобшение теоремы Бялого для $n=3$, а при $n=4,5,6$ аналогичный результат доказан при некоторых дополнительных предположениях о спектре потенциала. 


\section{§2. Вспомогательные утверждения}

Итак, рассмотрим гамильтонову систему, конфигурационньм пространством которой является двумерньй тор $\mathbb{T}^{2}=\left\{q_{1}, q_{2} \bmod 2 \pi\right\}$. Функция Гамильтона имеет вид

$$
H=\frac{a p_{1}^{2}+2 b p_{1} p_{2}+c p_{2}^{2}}{2}+W\left(q_{1}, q_{2}\right),
$$

где потенциал $W-2 \pi$-периодическая функция по $q_{1}$ и $q_{2} ; a, b$ и $c$ - вешественные постоянные, причем $a>0, a c-b^{2}>0$. Будем искать потенциал $W$, при котором существует полиномиальный по импульсам интеграл $n$-й степени $F$ с $2 \pi$-периодическими коэффициентами, независимый от интеграла энергии $H$.

Пусть сумма

$$
W=\sum_{-\infty}^{+\infty}[W]_{w_{1} w_{2}} e^{i\left(w_{1} q_{1}+w_{2} q_{2}\right)}
$$

будет рядом Фурье функции $W$. Спектром функции $W$ назовем (вообше говоря, бесконечное) подмножество целочисленной решетки

$$
S=\left\{w=\left(w_{1}, w_{2}\right) \in \mathbb{Z}^{2}:[W]_{w_{1} w_{2}} \neq 0\right\} .
$$

Оно переходит в себя при отражении $w \rightarrow-w$.

Пусть имеется полиномиальньй по импульсам интеграл $n$-й степени

$$
F=F_{n}+F_{n-1}+F_{n-2}+\cdots+F_{0}
$$

где $F_{k}$ - однородньй полином по импульсам степени $k$, коэффициенты которого являются гладкими функциями от $q_{1}$ и $q_{2}$. Заметим, что скобка Пуассона двух однородных полиномов по $p_{1}, p_{2}$ степени $r$ и $s$ будет однородным полиномом степени $r+s-1$. Отсюда вытекает следующий факт: если $F$ - интеграл гамильтоновой системы с гамильтонианом (2.1), то функции

$$
\Phi_{1}=F_{n}+F_{n-2}+\cdots, \quad \Phi_{2}=F_{n-1}+F_{n-3}+\cdots
$$

также будут интегралами этой системы. Поэтому будем считать $F$ суммой однородных полиномов только четных или только нечетных степеней.

Условия сушествования линейных и квадратичных интегралов хорошо известны [1]-[3]. При $n=1$ спектр потенциала $W$ лежит на одной прямой, проходящей через начало координат. При $n=2$ спектр лежит на двух прямых, ортогональных во внутренней метрике, порожденной кинетической энергией. Отметим, что для метрик обшего вида такая ситуация не типична: нет двух ортогональных прямых, проходящих через точки целочисленной решетки.

Пусть спектр потенциала лежит на одной прямой. Тогда $W=f\left(m q_{1}+n q_{2}\right)$, где $m, n$-взаимно простые целые числа, а $f-2 \pi$-периодическая функция. В этом случае имеется линейный по импульсам интеграл $F_{1}=m p_{2}-n p_{1}$.

Пусть теперь спектр $W$ лежит на двух прямых, ортогонально пересекаюшихся в начале координат. Тогда

$$
W=f_{1}\left(m_{1} q_{1}+n_{1} q_{2}\right)+f_{2}\left(m_{2} q_{1}+n_{2} q_{2}\right),
$$


условие ортогональности имеет вид

$$
a m_{1} m_{2}+b\left(m_{1} n_{2}+m_{2} n_{1}\right)+c n_{1} n_{2}=0,
$$

а функции $f_{1}$ и $f_{2}$, разумеется, $2 \pi$-периодические. В этом случае имеется квадратичньй интеграл

$$
\begin{aligned}
F_{2}+F_{0}=\left(a\left(r_{1}+r_{2}\right)+2 b\right) & p_{1}^{2}+2\left(c-a r_{1} r_{2}\right) p_{1} p_{2} \\
& -\left(c\left(r_{1}+r_{2}\right)+2 b r_{1} r_{2}\right) p_{2}^{2}+2\left(r_{1}-r_{2}\right)\left(f_{1}-f_{2}\right)
\end{aligned}
$$

где $r_{i}=m_{i} / n_{i}$.

Пусть натуральная механическая система (2.1) допускает дополнительный независимьй интеграл $F$, степень которого $n \geqslant 3$. Если $n$ четное, то

$$
F=F_{n}+F_{n-2}+\cdots+F_{2}+F_{0}
$$

где $F_{0}$ является функцией от $q_{1}$ и $q_{2}$. Если $n$ нечетное, то

$$
F=F_{n}+F_{n-2}+\cdots+F_{3}+F_{1}
$$

Кинетическую энергию удобно привести к диагональному виду с помошью линейного преобразования

$$
\left(\begin{array}{l}
x_{1} \\
x_{2}
\end{array}\right)=\Gamma^{T}\left(\begin{array}{l}
q_{1} \\
q_{2}
\end{array}\right), \quad|\Gamma| \neq 0 .
$$

Перейдем к новьм переменным $x_{1}$ и $x_{2}$, которые являются конфформньми координатами на накрываюшей тор плоскости. Расширим это преобразование до канонического $q, p \rightarrow x, y$, где

$$
\left(\begin{array}{l}
y_{1} \\
y_{2}
\end{array}\right)=\Gamma^{-1}\left(\begin{array}{l}
p_{1} \\
p_{2}
\end{array}\right)
$$

Тогда

$$
\begin{gathered}
H=H_{2}+V\left(x_{1}, x_{2}\right), \quad H_{2}=\frac{y_{1}^{2}+y_{2}^{2}}{2} \\
V=\sum_{-\infty}^{+\infty}[W]_{w} e^{i\left(\Gamma^{-1} w, x\right)}=\sum_{-\infty}^{+\infty}[W]_{w} e^{i(w, q)}, \quad w \in \mathbb{Z}^{2}
\end{gathered}
$$

Ясно, что в обшем случае $\Gamma^{-1} w \notin \mathbb{Z}^{2}$. После указанной подстановки спектр потенциала $W$ будет лежать в узлах решетки параллелограммов.

Пусть

$$
\begin{aligned}
F_{n} & =\sum_{i=0}^{n} b_{i}^{[n]} y_{1}^{n-i} y_{2}^{i}, \\
F_{n-2} & =\sum_{i=0}^{n-2} b_{i}^{[n-2]} y_{1}^{n-2-i} y_{2}^{i}, \\
F_{n-4} & =\sum_{i=0}^{n-4} b_{i}^{[n-4]} y_{1}^{n-4-i} y_{2}^{i}, \ldots
\end{aligned}
$$


Как показал Пуанкаре ([14], см. также [11]), функции $F_{n}$ и $H_{2}$ можно считать независимьми. В противном случае найдется интеграл меньшей степени. Как показано в [4], функции $F_{n}$ и $H_{2}$ зависимы тогда и только тогда, когда

$$
b_{0}^{[n]}-b_{2}^{[n]}+b_{4}^{[n]}-\cdots=0, \quad b_{1}^{[n]}-b_{3}^{[n]}+b_{5}^{[n]}-\cdots=0 .
$$

Суммы в левых частях этих равенств будем называть суммами Биркгофа (см. [1]). Они получаются как действительная и мнимая части $F_{n}$, если положить $y_{1}=1$ и $y_{2}=i$.

С помощью метода Пуанкаре доказываются следующие утверждения (см. [11; гл. IV]). Во-первых, коэффициенты $b_{i}^{[n]}$ старшего полинома $F_{n}$ постоянны (не зависят от координат $x_{1}, x_{2}$ ). Далее, пусть спектр потенциала содержит точку с координатами $m_{1}, m_{2}$, причем $m_{1}^{2}+m_{2}^{2} \neq 0$. Тогда

$$
y_{2} \frac{\partial F_{n}}{\partial y_{1}}-y_{1} \frac{\partial F_{n}}{\partial y_{2}}=0, \text { если } m_{1} y_{1}+m_{2} y_{2}=0 .
$$

Эквивалентное утверждение: многочлен

$$
m_{1} \frac{\partial F_{n}}{\partial y_{1}}+m_{2} \frac{\partial F_{n}}{\partial y_{2}}
$$

делится нацело на $m_{1} y_{1}+m_{2} y_{2}$. Частное от деления этих многочленов обозначим $G_{m_{1} m_{2}} ;$ это - однородньй многочлен степени $n-2$.

Наш основной результат составляет

Теорема 1. На прямой $m_{1} y_{1}+m_{2} y_{2}=0$ справедливо равенство

$$
m_{1} \frac{\partial G_{m_{1} m_{2}}}{\partial y_{1}}+m_{2} \frac{\partial G_{m_{1} m_{2}}}{\partial y_{2}}=0
$$

Это утверждение дополняет классический результат Пуанкаре (2.4). Чтобы лучше понять смысл условий (2.4) и (2.5), рассмотрим частный случай, когда точки спектра потенциала лежат на вертикальной прямой $\left(m_{1}=0\right)$. Тогда условие Пуанкаре (2.4) эквивалентно условию $b_{1}^{[n]}=0$, а условие (2.5) переходит в равенство $b_{3}^{[n]}=0$.

ЗАмЕчАнИЕ. Естественно предположить, что справедливо более общее утверждение о равенстве нулю коэффициентов многочлена $F_{n}$ со всеми нечетными номерами. K сожалению, это утверждение нам не удалось доказать. Однако оно все равно не позволило бы дать полное доказательство гипотезы о неприводимых интегралах, о которой говорилось во введении (см. §5). 


\section{§3. Доказательство основной теоремы}

Из уравнения $\{F, H\}=0$ получим соотношения на коэффициенты интеграла $F$. Выпишем группу уравнений при мономах $y_{1}^{i} y_{2}^{j}$, где $i+j=n+1$. Она состоит из $n+2$ уравнений:

$$
\frac{\partial b_{j}^{[n]}}{\partial x_{1}}+\frac{\partial b_{j-1}^{[n]}}{\partial x_{2}}=0, \quad j=0, \ldots, n+1, \quad b_{-1}^{[n]}=b_{n+1}^{[n]}=0 .
$$

Как уже говорилось, решениями данных уравнений являются постоянные; пусть

$$
b_{i}^{[n]}=a_{i}^{[n]}=\text { const }, \quad i=0, \ldots, n .
$$

Приравнивая нулю коэффициенты при разных мономах $y_{1}^{i} y_{2}^{j}$, где $i+j=n-1$, получим следуюшую группу $n$ уравнений

$$
\begin{gathered}
\frac{\partial b_{j}^{[n-2]}}{\partial x_{1}}+\frac{\partial b_{j-1}^{[n-2]}}{\partial x_{2}}=(n-j) a_{j}^{[n]} \frac{\partial V}{\partial x_{1}}+(j+1) a_{j+1}^{[n]} \frac{\partial V}{\partial x_{2}} \\
j=0, \ldots, n-1, \quad b_{-1}^{[n-2]}=b_{n-1}^{[n-2]}=0 .
\end{gathered}
$$

Аналогично выглядят остальные уравнения

$$
\begin{aligned}
& \frac{\partial b_{j}^{[n-4]}}{\partial x_{1}}+\frac{\partial b_{j-1}^{[n-4]}}{\partial x_{2}}=(n-2-j) b_{j}^{[n-2]} \frac{\partial V}{\partial x_{1}}+(j+1) b_{j+1}^{[n-2]} \frac{\partial V}{\partial x_{2}} \\
& j=0, \ldots, n-3, \quad b_{-1}^{[n-4]}=b_{n-3}^{[n-4]}=0 ; \\
& \frac{\partial b_{j}^{[n-2 l]}}{\partial x_{1}}+\frac{\partial b_{j-1}^{[n-2 l]}}{\partial x_{2}}=(n-2(l-1)-j) b_{j}^{[n-2(l-1)]} \frac{\partial V}{\partial x_{1}}+(j+1) b_{j+1}^{[n-2(l-1)]} \frac{\partial V}{\partial x_{2}} \\
& j=0, \ldots, n-2 l+1, \quad b_{-1}^{[n-2 l]}=b_{n-2 l+1}^{[n-2 l]}=0 ; \\
& (3.1 . n-2 l)
\end{aligned}
$$

Далее, если $n$ четное, то последняя группа состоит из двух уравнений:

$$
\frac{\partial b_{0}^{[0]}}{\partial x_{1}}=2 b_{0}^{[2]} \frac{\partial V}{\partial x_{1}}+b_{1}^{[2]} \frac{\partial V}{\partial x_{2}}, \quad \frac{\partial b_{0}^{[0]}}{\partial x_{2}}=b_{1}^{[2]} \frac{\partial V}{\partial x_{1}}+2 b_{2}^{[2]} \frac{\partial V}{\partial x_{2}} .
$$

Если $n$ нечетное, то последнее уравнение имеет вид

$$
0=b_{0}^{[1]} \frac{\partial V}{\partial x_{1}}+b_{1}^{[1]} \frac{\partial V}{\partial x_{2}} .
$$

Уравнения (3.1.n - 2) являются линейными; решим их методом Фурье. Пусть

$$
V=\sum_{-\infty}^{+\infty}[V]_{u v} e^{i\left(u x_{1}+v x_{2}\right)}, \quad b_{j}^{[n-2]}=\sum_{-\infty}^{+\infty}\left[b_{j}^{[n-2]}\right]_{u v} e^{i\left(u x_{1}+v x_{2}\right)}, \quad j=0, \ldots, n-2,
$$


где $[V]_{u v},\left[b_{j}^{[n-2]}\right]_{u v}-$ коэффициенты Фурье. Нетрудно показать, что для каждого набора $u, v$ имеем систему

$$
\left(\begin{array}{ccccccc}
-u & 0 & 0 & \ldots & 0 & 0 & n a_{0}^{[n]} u+a_{1}^{[n]} v \\
-v & -u & 0 & \ldots & 0 & 0 & (n-1) a_{1}^{[n]} u+2 a_{2}^{[n]} v \\
0 & -v & -u & \ldots & 0 & 0 & (n-2) a_{2}^{[n]} u+3 a_{3}^{[n]} v \\
\ldots & \ldots & \ldots & \ldots \ldots \\
0 & 0 & 0 & \ldots & -v & -u & 2 a_{n-2}^{[n]} u+(n-1) a_{n-1}^{[n]} v \\
0 & 0 & 0 & \ldots & 0 & -v & a_{n-1}^{[n]} u+n a_{n}^{[n]} v
\end{array}\right)\left(\begin{array}{c}
{\left[b_{0}^{[n-2]}\right] u v} \\
{\left[b_{1}^{[n-2]}\right]_{u v}} \\
{\left[b_{2}^{[n-2]}\right]_{u v}} \\
\ldots \\
{\left[b_{n-2}^{[n-2]}\right]_{u v}} \\
{[V] u v}
\end{array}\right)=0
$$

Система (3.2) имеет нетривиальное решение только для тех $u$ и $v$, для которых определитель системы равен нулю. Подсчитаем определитель $(n \times n)$-матрицы, раскладывая его по последнему столбцу:

$$
\begin{aligned}
0 & =\left(n a_{0}^{[n]} u+a_{1}^{[n]} v\right) v^{n-1}-\left((n-1) a_{1}^{[n]} u+2 a_{2}^{[n]} v\right) u v^{n-2} \\
& +\cdots+(-1)^{n}\left(2 a_{n-2}^{[n]} u+(n-1) a_{n-1}^{[n]} v\right) u^{n-2} v+(-1)^{n-1}\left(a_{n-1}^{[n]} u+n a_{n}^{[n]} v\right) u^{n-1} .
\end{aligned}
$$

Перепишем это тождество. Пусть $z=u / v$; тогда $z$-корень многочлена степени $n$ :

$$
\sum_{i=0}^{n}(-1)^{i}\left((i+1) a_{i+1}^{[n]}-(n-i+1) a_{i-1}^{[n]}\right) z^{i}=0
$$

При этом константы $a_{j}^{[n]}$ (причем $\left.a_{-1}^{[n]}=a_{n+1}^{[n]}=0\right)$ должны быть такими, чтобы уравнение (3.3) допускало хотя бы один вешественный корень $z_{m}=u_{m} / v_{m}$. Пусть функция $V$ имеет вид

$$
V=\sum_{m=1}^{n} f_{m}\left(u_{m} x_{1}+v_{m} x_{2}\right)
$$

где

$$
f_{m}=\sum_{\lambda=-\infty}^{+\infty}[V]_{\lambda u_{m}, \lambda v_{m}} e^{i \lambda\left(u_{m} x_{1}+v_{m} x_{2}\right)}
$$

т.е. спектр функции $V$ лежит самое большее на $n$ прямых в вещественной плоскости. Прямые, которые содержат спектр функции $V$, будем назьвать прямыми спектра функции $V$. Возьмем, например, $k$-ю прямую. С помощью поворота плоскости вокруг начала координат эту прямую можно сделать вертикальной. Расширяя это преобразование до канонического, получим функцию Гамильтона вида (2.2), однако теперь потенциальная энергия принимает форму

$$
V=f_{k}\left(v_{k} x_{2}\right)+\sum_{\substack{m=1 \\ m \neq k}}^{n} f_{m}\left(u_{m} x_{1}+v_{m} x_{2}\right) .
$$

Заметим, что

$$
\frac{\partial f_{m}}{\partial x_{2}}=v_{m} f_{m}^{\prime}=\frac{v_{m}}{u_{m}} \frac{\partial f_{m}}{\partial x_{1}}, \quad m=1, \ldots, n, \quad m \neq k .
$$

Штрих означает производную по своему аргументу. 
Из трех первых уравнений $(3.1 . n-2)$ найдем функции $b_{0}^{[n-2]}$ и $b_{1}^{[n-2]}$. Для этого выпишем явно три первых уравнения из группы уравнений $(3.1 . n-2)$, где $a_{1}^{[n]}=0$ :

$$
\begin{gathered}
\frac{\partial b_{0}^{[n-2]}}{\partial x_{1}}=n a_{0}^{[n]} \frac{\partial V}{\partial x_{1}}, \\
\frac{\partial b_{1}^{[n-2]}}{\partial x_{1}}+\frac{\partial b_{0}^{[n-2]}}{\partial x_{2}}=2 a_{2}^{[n]} \frac{\partial V}{\partial x_{2}}, \\
\frac{\partial b_{2}^{[n-2]}}{\partial x_{1}}+\frac{\partial b_{1}^{[n-2]}}{\partial x_{2}}=(n-2) a_{2}^{[n]} \frac{\partial V}{\partial x_{1}}+3 a_{3}^{[n]} \frac{\partial V}{\partial x_{2}} .
\end{gathered}
$$

Из уравнения (3.5) выразим функцию $b_{0}^{[n-2]}$ :

$$
b_{0}^{[n-2]}=n a_{0}^{[n]} V+c_{0}^{[n-2]}\left(x_{2}\right)
$$

где функция $c_{0}^{[n-2]}$ зависит только от $x_{2}$. Найдем ее из уравнения $(3.6)$ :

$$
\frac{\partial b_{1}^{[n-2]}}{\partial x_{1}}+n a_{0}^{[n]}\left(\frac{d f_{k}}{d x_{2}}+\sum_{\substack{m=1 \\ m \neq k}}^{n} \frac{v_{m}}{u_{m}} \frac{\partial f_{m}}{\partial x_{1}}\right)+\frac{d c_{0}^{[n-2]}}{d x_{2}}=2 a_{2}^{[n]}\left(\frac{d f_{k}}{d x_{2}}+\sum_{\substack{m=1 \\ m \neq k}}^{n} \frac{v_{m}}{u_{m}} \frac{\partial f_{m}}{\partial x_{1}}\right) .
$$

Это уравнение имеет следующий вид:

$$
\frac{\partial \Delta}{\partial x_{1}}+\left(\frac{d c_{0}^{[n-2]}}{d x_{2}}-\left(2 a_{2}^{[n]}-n a_{0}^{[n]}\right) \frac{d f_{k}}{d x_{2}}\right)=0
$$

где

$$
\Delta=b_{1}^{[n-2]}-\left(2 a_{2}^{[n]}-n a_{0}^{[n]}\right)\left(\sum_{\substack{m=1 \\ m \neq k}}^{n} \frac{v_{m}}{u_{m}} f_{m}\right)
$$

Из уравнения (3.8) видно, что гармоники вида $e^{i \nu x_{2}}$ имеются только у функций $c_{0}^{[n-2]}$ и $f_{k}^{\prime}$. Для этих функций имеем уравнение

$$
\frac{d c_{0}^{[n-2]}}{d x_{2}}-\left(2 a_{2}^{[n]}-n a_{0}^{[n]}\right) \frac{d f_{k}}{d x_{2}}=0
$$

Отсюда находим функцию $c_{0}^{[n-2]}$ :

$$
c_{0}^{[n-2]}=\left(2 a_{2}^{[n]}-n a_{0}^{[n]}\right) f_{k}+a_{0}^{[n-2]}
$$

где $a_{0}^{[n-2]}=$ const. Итак, мы нашли функцию $b_{0}^{[n-2]}$ :

$$
b_{0}^{[n-2]}=n a_{0}^{[n]} V+\left(2 a_{2}^{[n]}-n a_{0}^{[n]}\right) f_{k}+a_{0}^{[n-2]} .
$$


Из уравнения (3.6) найдем функцию $b_{1}^{[n-2]}$. Вспомним, что нам удалось преобразовать уравнение (3.6) к виду (3.8), где

$$
\frac{\partial \Delta}{\partial x_{1}}=0
$$

Тогда

$$
b_{1}^{[n-2]}=\left(2 a_{2}^{[n]}-n a_{0}^{[n]}\right)\left(\sum_{\substack{m=1 \\ m \neq k}}^{n} \frac{v_{m}}{u_{m}} f_{m}\right)+c_{1}^{[n-2]}\left(x_{2}\right),
$$

где функция $c_{1}^{[n-2]}$ зависит только от $x_{2}$. Найдем ее из уравнения $(3.7)$, переписав его в явной форме:

$$
\begin{aligned}
\frac{\partial b_{2}^{[n-2]}}{\partial x_{1}}+\left(2 a_{2}^{[n]}-n a_{0}^{[n]}\right) & \left(\sum_{\substack{m=1 \\
m \neq k}}^{n}\left(\frac{v_{m}}{u_{m}}\right)^{2} \frac{\partial f_{m}}{\partial x_{1}}\right)+\frac{d c_{1}^{[n-2]}}{d x_{2}} \\
& =(n-2) a_{2}^{[n]} \frac{\partial V}{\partial x_{1}}+3 a_{3}^{[n]}\left(\frac{d f_{k}}{d x_{2}}+\sum_{\substack{m=1 \\
m \neq k}}^{n} \frac{v_{m}}{u_{m}} \frac{\partial f_{m}}{\partial x_{1}}\right) .
\end{aligned}
$$

Это уравнение имеет следующий вид:

$$
\frac{\partial \Upsilon}{\partial x_{1}}+\left(\frac{d c_{1}^{[n-2]}}{d x_{2}}-3 a_{3}^{[n]} \frac{d f_{k}}{d x_{2}}\right)=0
$$

где

$$
\Upsilon=b_{2}^{[n-2]}+\left(2 a_{2}^{[n]}-n a_{0}^{[n]}\right)\left(\sum_{\substack{m=1 \\ m \neq k}}^{n}\left(\frac{v_{m}}{u_{m}}\right)^{2} f_{m}\right)-(n-2) a_{2}^{[n]} V-3 a_{3}^{[n]}\left(\sum_{\substack{m=1 \\ m \neq k}}^{n} \frac{v_{m}}{u_{m}} f_{m}\right) .
$$

Из уравнения (3.10) видно, что гармоники вида $e^{i \nu x_{2}}$ имеются только у функций $c_{1}^{[n-2]}$ и $f_{k}^{\prime}$. Для этих функций имеем уравнение

$$
\frac{d c_{0}^{[n-2]}}{d x_{2}}-3 a_{3}^{[n]} \frac{d f_{k}}{d x_{2}}=0
$$

Отсюда находим функцию $c_{1}^{[n-2]}$ :

$$
c_{1}^{[n-2]}=3 a_{3}^{[n]} f_{k}+a_{1}^{[n-2]},
$$

где $a_{1}^{[n-2]}=$ const. Итак, мы нашли функцию $b_{1}^{[n-2]}$ :

$$
b_{1}^{[n-2]}=3 a_{3}^{[n]} f_{k}+\left(2 a_{2}^{[n]}-n a_{0}^{[n]}\right)\left(\sum_{\substack{m=1 \\ m \neq k}}^{n} \frac{v_{m}}{u_{m}} f_{m}\right)+a_{1}^{[n-2]}
$$


Обратимся к первому уравнению группы $(3.1 . n-4)$. Подставляя в это уравнение вместо функций $b_{0}^{[n-2]}$ и $b_{1}^{[n-2]}$ их выражения $(3.9)$ и (3.11), получим

$$
\begin{aligned}
& \frac{\partial b_{0}^{[n-4]}}{\partial x_{1}}=(n-2)\left(n a_{0}^{[n]} V+\left(2 a_{2}^{[n]}-n a_{0}^{[n]}\right) f_{k}+a_{0}^{[n-2]}\right) \frac{\partial V}{\partial x_{1}} \\
& +\left(3 a_{3}^{[n]} f_{k}+\left(2 a_{2}^{[n]}-n a_{0}^{[n]}\right) \sum_{\substack{m=1 \\
m \neq k}}^{n} \frac{v_{m}}{u_{m}} f_{m}+a_{1}^{[n-2]}\right)\left(\frac{d f_{k}}{d x_{2}}+\sum_{\substack{m=1 \\
m \neq k}}^{n} \frac{v_{m}}{u_{m}} \frac{\partial f_{m}}{\partial x_{1}}\right) \\
& =n(n-2) a_{0}^{[n]} \frac{1}{2} \frac{\partial V^{2}}{\partial x_{1}}+(n-2)\left(2 a_{2}^{[n]}-n a_{0}^{[n]}\right) \frac{\partial f_{k} V}{\partial x_{1}}+(n-2) a_{0}^{[n-2]} \frac{\partial V}{\partial x_{1}} \\
& +\left(3 a_{3}^{[n]} f_{k}+a_{1}^{[n-2]}\right) \frac{d f_{k}}{d x_{2}}+\left(2 a_{2}^{[n]}-n a_{0}^{[n]}\right) \frac{d f_{k}}{d x_{2}}\left(\sum_{\substack{m=1 \\
m \neq k}}^{n} \frac{v_{m}}{u_{m}} f_{m}\right) \\
& \quad+3 a_{3}^{[n]} \sum_{\substack{m=1 \\
m \neq k}}^{n} \frac{v_{m}}{u_{m}} \frac{\partial f_{k} f_{m}}{\partial x_{1}}+\left(2 a_{2}^{[n]}-n a_{0}^{[n]}\right) \frac{1}{2} \frac{\partial}{\partial x_{1}}\left(\left(\sum_{m=1}^{n} \frac{v_{m}}{u_{m}} f_{m}\right)^{2}\right) \\
& \quad+a_{1}^{[n-2]} \sum_{\substack{m=1 \\
m \neq k}}^{n} \frac{v_{m}}{u_{m}} \frac{\partial f_{m}}{\partial x_{1}} .
\end{aligned}
$$

Это уравнение имеет следующий вид:

$$
\frac{\partial \Psi}{\partial x_{1}}+\left(3 a_{3}^{[n]} f_{k}+a_{1}^{[n-2]}\right) \frac{d f_{k}}{d x_{2}}+\left(2 a_{2}^{[n]}-n a_{0}^{[n]}\right) \frac{d f_{k}}{d x_{2}}\left(\sum_{\substack{m=1 \\ m \neq k}}^{n} \frac{v_{m}}{u_{m}} f_{m}\right)=0
$$

где

$$
\begin{aligned}
\Psi & =\frac{n(n-2)}{2} a_{0}^{[n]} V^{2}+(n-2)\left(2 a_{2}^{[n]}-n a_{0}^{[n]}\right) f_{k} V+(n-2) a_{0}^{[n-2]} V \\
& +\left(3 a_{3}^{[n]} f_{k}+a_{1}^{[n-2]}\right)\left(\sum_{\substack{m=1 \\
m \neq k}}^{n} \frac{v_{m}}{u_{m}} f_{m}\right)+\frac{1}{2}\left(2 a_{2}^{[n]}-n a_{0}^{[n]}\right)\left(\sum_{\substack{m=1 \\
m \neq k}}^{n} \frac{v_{m}}{u_{m}} f_{m}\right)^{2}-b_{0}^{[n-4]} .
\end{aligned}
$$

Из уравнения (3.12) видно, что гармоники вида $e^{i \nu x_{2}}$ имеются только у функций $f_{k}^{\prime}, f_{k} f_{k}^{\prime}$. Для функции $f_{k}$ имеем уравнение

$$
\left(3 a_{3}^{[n]} f_{k}+a_{1}^{[n-2]}+\varkappa\right) v_{k} f_{k}^{\prime}=0,
$$

где $\varkappa$ - среднее значение функции

$$
\left(2 a_{2}^{[n]}-n a_{0}^{[n]}\right)\left(\sum_{\substack{m=1 \\ m \neq k}}^{n} \frac{v_{m}}{u_{m}} f_{m}\right)
$$

По предположению $f_{k}$ - непостоянная аналитическая функция. Поскольку в кольце аналитических функций нет делителей нуля, то $a_{3}^{[n]}=0$. Что и требовалось доказать. 


\section{§4. Интегралы третьей степени}

ТЕОРЕма 2. Если гамильтонова система с гамильтонианом (2.1) допускает интеграл третьей степени $F_{3}+F_{1}$, причем $F_{3} \neq 0$, то спектр потенциальной әнергии лежст на одной прямой, проходящей через начало координат.

СлеДСтвИЕ. В предположении теоремь 2 уравнения Гамильтона имеют интеграл, линейньй по импульсам.

Если $a=c$ и $b=0$, то теорема 2 переходит в теорему М. Л. Бялого [8].

ДоКАЗАТЕЛЬСТво ТЕОРЕмЫ 2 . Поскольку $F_{3} \neq 0$, то алгебраическое уравнение (3.3) имеет не более трех вешественных корней. Таким образом, число прямых спектра потенциальной энергии не превосходит трех.

Случай, когда спектр лежит на двух прямых, рассматривался в [11; гл. IV]. Показано, что уравнения Гамильтона допускают дополнительный полиномиальньй интеграл тогда и только тогда, когда эти прямые ортогональны (в метрике, определяемой кинетической энергией). Подчеркнем, что это утверждение справедливо для интегралов любой степени. Если прямые спектра ортогональны, то имеется полиномиальный интеграл второй степени. Нам остается показать, что в этом случае нет нетривиальных интегралов степени 3 .

Предположим противное: пусть имеется интеграл третьей степени $F_{3}+F_{1}$, где

$$
F_{3}=a_{0} y_{1}^{3}+a_{1} y_{1}^{2} y_{2}+a_{2} y_{1} y_{2}^{2}+a_{3} y_{2}^{3} .
$$

С помощью поворота одну из прямых спектра можно сделать вертикальной. Тогда другая прямая (ввиду ортогональности) будет горизонтальной. Согласно (2.4) $a_{1}=a_{2}=0$. Применяя теорему 1 , получаем еше два соотношения: $a_{0}=a_{3}=0$. Следовательно, $F_{3} \equiv 0$. Получили противоречие.

ЗАмЕчАниЕ. Случай, когда спектр лежит на двух прямых, в работе [8] не рассмотрен, вероятно, по причине недостатка места.

Итак, осталось рассмотреть случай, когда имеется три различных прямых спектра. Одну из этих прямых считаем вертикальной. Тогда в (4.1) $a_{1}=a_{3}=0$. Пусть $(u, v)$ - еше одна точка спектра, причем $u \neq 0$. Соотношения $(2.4)$ и (2.5) приводят к двум равенствам:

$$
3 a_{0} v^{2}+a_{2}\left(u^{2}-2 v^{2}\right)=0, \quad a_{0} u^{2}+a_{2} v^{2}=0 .
$$

Поскольку числа $a_{0}$ и $a_{2}$ отличны от нуля (иначе $F_{3}=0$ ), то определитель этой линейной системы должен обратиться в нуль:

$$
3 v^{4}+2 u^{2} v^{2}-u^{4}=0 \text { или } 3 z^{4}+2 z^{2}-1=0,
$$

где $z=v / u$. Корни этого биквадратного уравнения равны $\pm \sqrt{3} / 3$. Таким образом, углы между прямыми спектра равны $\pi / 3$. При этом каждое из уравнений (4.2) приводит к следуюшему соотношению на оставшиеся коэффициенты $a_{0}$ и $a_{2}$ :

$$
3 a_{0}+a_{2}=0
$$


ЗАмечАниЕ. Именно этот случай выпал из рассмотрения в работе [8]. М. Л. Бяльй вводит числа

$$
k_{i i}=u_{i}\left(a_{1}+3 a_{3}+z_{i}\left(3 a_{0}+a_{2}\right)\right),
$$

где $z_{i}=v_{i} / u_{i}-$ корни многочлена (3.3), и считает, что только одно из них может быть равно нулю. Однако в выделенном нами случае $k_{i i}=0$ для всех $1 \leqslant i \leqslant 3$. Правда, в [8] рассматривается метрика стандартного вида (1.2), и поэтому (ввиду иррациональности корней биквадратного уравнения (4.3)) наклонные прямые спектра на самом деле не могут проходить через точки целочисленной решетки.

Итак, осталось рассмотреть исключительный случай, когда три прямые спектра потенциальной энергии образуют между собой углы, кратные $\pi / 3$. Функция Гамильтона приводится к виду

$$
H=\frac{\left(y_{1}^{2}+y_{2}^{2}\right)}{2}+f_{1}\left(\sqrt{3} x_{1}+x_{2}\right)+f_{2}\left(x_{2}\right)+f_{3}\left(-\sqrt{3} x_{1}+x_{2}\right),
$$

где $f_{s}$ - непостоянные периодические аналитические функции.

Дополнительный интеграл третьей степени $F$ имеет вид $F_{3}+b_{0} y_{1}+b_{1} y_{2}$, где $b_{0}$ и $b_{1}-$ аналитические функции на конфигурационном торе. В нашем случае $a_{1}=$ $a_{3}=0$ и выполнено соотношение (4.4). Поскольку $F_{3} \neq 0$, то можно положить $a_{0}=1$ и $a_{2}=-3$.

Функции $f_{s}$ определены с точностью до произвольных аддитивных постоянных. Будем считать, что $\left\langle f_{2}\right\rangle=0$, где $\langle\cdot\rangle$ - среднее функции по тору $\mathbb{T}^{2}=$ $\left\{x_{1}, x_{2} \bmod 2 \pi\right\}$. Поскольку $f_{2}-$ периодическая функция, то найдется такое $x_{2}^{0}$, что $f_{2}^{\prime}\left(x_{2}^{0}\right)=0$. Положим

$$
\left\langle f_{1}\right\rangle=-\left\langle f_{3}\right\rangle, \quad \frac{\left\langle f_{3}\right\rangle}{\sqrt{3}}=-f_{2}\left(x_{2}^{0}\right)+\frac{\left\langle b_{0}\right\rangle}{6} .
$$

Условие $\{H, F\}=0$ эквивалентно следующей системе уравнений для $b_{0}$ и $b_{1}$ :

$$
\begin{aligned}
& \frac{\partial b_{0}}{\partial x_{1}}+3 \sqrt{3}\left(f_{3}^{\prime}-f_{1}^{\prime}\right)=0, \quad \frac{\partial b_{0}}{\partial x_{2}}+\frac{\partial b_{1}}{\partial x_{1}}+6\left(f_{1}^{\prime}+f_{2}^{\prime}+f_{3}^{\prime}\right)=0, \\
& \frac{\partial b_{1}}{\partial x_{2}}-3 \sqrt{3}\left(f_{3}^{\prime}-f_{1}^{\prime}\right)=0, \quad \sqrt{3} b_{0}\left(f_{3}^{\prime}-f_{1}^{\prime}\right)-b_{1}\left(f_{1}^{\prime}+f_{2}^{\prime}+f_{3}^{\prime}\right)=0 .
\end{aligned}
$$

Первое и третье уравнения дают равенства

$$
b_{0}=3\left[f_{1}+f_{3}+a_{0}\left(x_{2}\right)\right], \quad b_{1}=3 \sqrt{3}\left[f_{3}-f_{1}+a_{1}\left(x_{1}\right)\right],
$$

где $a_{0}$ и $a_{1}-$ пока неизвестные периодические функции одной переменной. Подставляя (4.7) во второе уравнение (4.6), получаем равенство

$$
\frac{d a_{0}}{d x_{2}}+\sqrt{3} \frac{d a_{1}}{d x_{1}}+2 f_{2}^{\prime}=0
$$

Усредняя это равенство по $x_{1}$ и $x_{2}$, получаем, что $a_{0}=-2 f_{2}+c_{0}, c_{0}=$ const, и $a_{1}=c_{1}=$ const. 
Итак, равенства (4.7) принимают следующий вид:

$$
b_{0}=3\left(f_{1}-2 f_{2}+f_{3}+c_{0}\right), \quad b_{1}=3 \sqrt{3}\left(f_{3}-f_{1}+c_{1}\right) .
$$

Подставляя (4.8) в последнее уравнение (4.6), получаем нетривиальное соотношение для функций $f_{s}$ :

$$
2 f_{1}^{\prime}\left(f_{2}-f_{3}-\frac{c_{0}}{2}\right)+f_{2}^{\prime}\left(f_{1}-f_{3}-c_{1}\right)+2 f_{3}^{\prime}\left(f_{1}-f_{2}+\frac{c_{0}}{2}-\frac{c_{1}}{2}\right)=0
$$

Положим $x_{2}=x_{2}^{0}$ и

$$
\begin{aligned}
& g_{1}\left(x_{1}\right)=f_{1}\left(\sqrt{3} x_{1}+x_{2}^{0}\right)-f_{2}\left(x_{2}^{0}\right)+\frac{c_{0}}{2}-\frac{c_{1}}{2}, \\
& g_{3}\left(x_{1}\right)=f_{3}\left(-\sqrt{3} x_{1}+x_{2}^{0}\right)-f_{2}\left(x_{2}^{0}\right)+\frac{c_{0}}{2} .
\end{aligned}
$$

Поскольку $x_{2}^{0}$ - критическая точка функции $f_{2}$, то равенство (4.9) принимает вид

$$
\frac{\partial}{\partial x_{1}}\left(g_{1} g_{3}\right)=0 \text {. }
$$

Следовательно,

$$
g_{1} g_{3}=c=\text { const } .
$$

Усредняя первое равенство (4.8) по $\mathbb{T}^{2}$, получаем

$$
\left\langle b_{0}\right\rangle=3\left(\left\langle f_{1}\right\rangle-2\left\langle f_{2}\right\rangle+\left\langle f_{3}\right\rangle+c_{0}\right)
$$

Согласно предположениям $\left\langle f_{1}\right\rangle+\left\langle f_{3}\right\rangle=0$ (см. (4.5)) и $\left\langle f_{2}\right\rangle=0$. Следовательно, $c_{0}=\left\langle b_{0}\right\rangle / 3$. Ясно, что

$$
\left\langle g_{3}\right\rangle=-\frac{\left\langle f_{3}\right\rangle}{\sqrt{3}}-f_{2}\left(x_{2}^{0}\right)+\frac{\left\langle b_{0}\right\rangle}{6} .
$$

Однако ввиду (4.5) правая часть этого равенства равна нулю. Поскольку $g_{3}-$ периодическая функция с нулевым средним, то она обязательно имеет нули. Следовательно, постоянная $c$ из равенства (4.11) равна нулю. Так как в кольце аналитических функций нет делителей нуля, то либо $g_{1}$, либо $g_{3}$ тождественно равны нулю. Но тогда согласно (4.10) одна из функций $f_{1}$ или $f_{3}$ будет постоянной. Таким образом, уравнение (4.9) не имеет нетривиальных периодических решений. Теорема доказана.

ЗАмЕчАниЕ. Непериодические решения уравнения (4.9) сушествуют. Вот простой пример:

$$
f_{1}=\exp \left(\sqrt{3} x_{1}+x_{2}\right), \quad f_{2}=\exp \left(-2 x_{2}\right), \quad f_{3}=\exp \left(-\sqrt{3} x_{1}+x_{2}\right)
$$

а постоянные $c_{0}$ и $c_{1}$ равны нулю. Этот случай отвечает интегрируемой цепочке Тоды для трех частиц после исключения центра масс. 


\section{§5. Интегралы четвертой степени}

Предположим, что уравнения Гамильтона с гамильтонианом (2.1) допускают интеграл четвертой степени $F_{4}+F_{2}+F_{0}$, где

$$
F_{4}=a_{0} y_{1}^{4}+a_{1} y_{1}^{3} y_{2}+a_{2} y_{1}^{2} y_{2}^{2}+a_{3} y_{1} y_{2}^{3}+a_{4} y_{2}^{4} .
$$

Без ушерба для общности можно считать, что одна из прямых спектра вертикальна. Тогда согласно результатам $\S 2$ имеют место равенства $a_{1}=a_{3}=0$.

Предположим, что точка $(u, v), u \neq 0$, есть точка спектра. Условия (2.4) и (2.5) дадут нам два соотношения:

$$
\begin{aligned}
& 2 a_{0} v^{3} u+a_{2}\left(u^{3} v-u v^{3}\right)-2 a_{4} u^{3} v=0 \\
& 2 a_{0} v u^{3}+a_{2}\left(u v^{3}-u^{3} v\right)-2 a_{4} u v^{3}=0
\end{aligned}
$$

Они заведомо выполнены, если $v=0$. Это означает, что наличие горизонтальной прямой в спектре не противоречит интегрируемости рассматриваемой системы.

Какие еше прямые могут быть прямыми спектра системы с интегралом четвертой степени? Сокращая левые части соотношений (5.2) на $u v$ и складывая их, получим

$$
2\left(a_{0}-a_{4}\right)\left(u^{2}+v^{2}\right)=0 .
$$

Отсюда $a_{0}=a_{4}$. После этого каждое из уравнений (5.2) можно привести к виду

$$
\left(2 a_{0}-a_{2}\right)\left(u^{2}-v^{2}\right)=0
$$

Следовательно, либо $2 a_{0}=a_{2}$, либо $u= \pm v$. В первом случае сумма Биркгофа $a_{0}-a_{2}+a_{4}$ равна нулю. Учитывая еше равенства $a_{1}=a_{3}=0$, получаем, что многочлен $F_{4}$ делится нацело на кинетическую энергию $\left(y_{1}^{2}+y_{2}^{2}\right) / 2$. Однако этот случай уже нами исключен из рассмотрения (см. $\S 2)$. Итак, $u= \pm v$. Это означает, что к "допустимьм" прямым спектра относятся также биссектрисы координатных углов.

Таким образом, доказано

ПРЕДЛОЖЕНИЕ 1. Если система с гамильтонианом (2.1) допускает неприводимый интеграл четвертой степени, то ее спектр лежит на трех или четырех прямых, образующих между собой угль $\pi / 4$ или $\pi / 2$.

Это утверждение оставляет нерассмотренной систему с гамильтонианом

$$
H=\frac{\left(y_{1}^{2}+y_{2}^{2}\right)}{2}+f_{1}\left(x_{1}\right)+f_{2}\left(x_{1}+x_{2}\right)+f_{3}\left(x_{2}\right)+f_{4}\left(x_{2}-x_{1}\right) .
$$

Здесь $f_{s}-2 \pi$-периодические функции, по крайней мере три из которых не постоянны. Интеграл четвертой степени, независимый от интеграла энергии, приводится к виду

$$
F=\frac{\left(y_{1}^{4}+y_{2}^{4}\right)}{4}+F_{2}+F_{0}
$$


Из условия $\{H, F\}=0$ можно получить (как в $\S 4$ ) соотношение для функций $f_{s}$, аналогичное (4.9):

$$
\begin{array}{r}
f_{1}^{\prime \prime} f_{2}+3 f_{1}^{\prime} f_{2}^{\prime}+2 f_{1} f_{2}^{\prime \prime}-f_{1}^{\prime \prime} f_{4}+3 f_{1}^{\prime} f_{4}^{\prime}-2 f_{1} f_{4}^{\prime \prime}-f_{2} f_{3}^{\prime \prime}-3 f_{2}^{\prime} f_{3}^{\prime}-2 f_{2}^{\prime \prime} f_{3} \\
+f_{3}^{\prime \prime} f_{4}+3 f_{3}^{\prime} f_{4}^{\prime}+2 f_{3} f_{4}^{\prime \prime}+c_{1}\left(f_{1}^{\prime \prime}-f_{3}^{\prime \prime}\right)+c_{2}\left(f_{2}^{\prime \prime}-f_{4}^{\prime \prime}\right)=0 .
\end{array}
$$

Здесь штрих обозначает производную по аргументу функции, $c_{1}$ и $c_{2}-$ некоторые постоянные. К сожалению, нам не удалось установить отсутствие нетривиальных периодических решений уравнения (5.3). Отметим, что (5.3) допускает непериодические решения.

\section{§6. Интегралы пятой и шестой степени}

Пусть теперь система с гамильтонианом (2.1) допускает интеграл 5-й степени $F_{5}+F_{3}+F_{1}$, причем

$$
F_{5}=a_{0} y_{1}^{5}+a_{2} y_{1}^{3} y_{2}^{2}+a_{4} y_{1} y_{2}^{4}+a_{5} y_{2}^{5} \text {. }
$$

Мы считаем, как обычно, что одна из прямых спектра вертикальна (поэтому в (6.1) уже положено $\left.a_{1}=a_{3}=0\right)$. Если этот интеграл неприводимый, то обязательно имеется еще хотя бы одна прямая спектра. Пусть она проходит через точку спектра с координатами $u, v(u \neq 0)$. Соотношения (2.4) и (2.5) дают нам два уравнения:

$$
\begin{gathered}
\gamma z^{4}+\delta z^{2}+a_{4}+5 a_{5} z=0 \\
6 \gamma z^{2}+\delta\left(3 z^{4}-2 z^{2}+1\right)+a_{4}\left(10 z^{2}+4\right)+30 a_{5} z^{3}=0 .
\end{gathered}
$$

Здесь $\gamma=5 a_{0}-2 a_{2}, \delta=3 a_{2}-4 a_{4}, z=v / u$.

На самом деле имеется еще одна прямая спектра, если интеграл неприводимый. Действительно, как показано в [11], если спектр лежит на двух прямых и эти прямые не ортогональные, то вообще нет дополнительных полиномиальных интегралов любой степени. Если же две прямые спектра ортогональны, то имеется квадратичный интеграл и поэтому исходный интеграл пятой степени оказывается приводимым.

Пусть третья прямая спектра проходит через точку с координатами $u_{1}, v_{1}$ $\left(u_{1} \neq 0\right)$ и $z_{1}=v_{1} / u_{1}$. Тогда $z_{1} \neq z$ удовлетворяет тем же самым уравнениям (6.2). Таким образом, мы имеем систему однородных линейных уравнений относительно $\gamma, \delta, a_{4}$ и $a_{5}$. Если определитель $\Delta$ этой системы отличен от нуля, то $F_{5}=0$ и поэтому интеграл оказывается приводимым.

Определитель $\Delta$ равен

$$
30\left(z^{2}+1\right)\left(z_{1}^{2}+1\right)\left(z-z_{1}\right)^{2}\left(z+z_{1}\right)\left[z^{2} z_{1}^{2}+\left(2 z z_{1}+1\right)^{2}-\left(2 z z_{1}+1\right)\left(z^{2}+z_{1}^{2}\right)\right] .
$$

Поскольку $z \neq z_{1}$, то он равен нулю в двух случаях:
a) $z_{1}=-z$,
b) $z^{2} z_{1}^{2}+\left(2 z z_{1}+1\right)^{2}-\left(2 z z_{1}+1\right)\left(z^{2}+z_{1}^{2}\right)=0$.

В случае а) дополнительные прямые спектра симметричны относительно первой вертикальной прямой. Уравнение случая b) легко решается, если положить 
$2 z z_{1}+1=x$. Тогда $x^{2}-x\left(z^{2}+z_{1}^{2}\right)+z^{2} z_{1}^{2}=0$. Отсюда $x=z^{2}$ или $x=z_{1}^{2}$. Следовательно,

$$
2 z z_{1}+1=z^{2} \text { или } 2 z z_{1}+1=z_{1}^{2} .
$$

В первом случае

$$
z_{1}=-\left(\frac{2 z}{1-z^{2}}\right)^{-1}
$$

Пусть вторая (третья) прямая спектра образует с горизонтальной осью угол $\varphi$ $\left(\varphi_{1}\right)$. Тогда соотношение (6.5) эквивалентно равенству

$$
\varphi_{1}=2 \varphi \pm \pi / 2
$$

Отсюда получаем простой способ построения третьей прямой спектра: вторую прямую поворачиваем на угол $\varphi$ против часовой стрелки и проводим перпендикулярную прямую. Аналогичный геометрический смысл имеет второе уравнение (6.4).

Отметим, что в качестве выделенной прямой спектра (которую мы считали вертикальной) можно взять любую из двух других прямых. Тогда взаимное расположение двух оставшихся прямых будет также удовлетворять указанным выше свойствам. В частности, пусть точки спектра лежат на вертикальной и горизонтальной прямой. Тогда эта прямая будет наклонена к горизонту под углом $\pi / 4$.

Пусть $l_{0}, l_{1}$ и $l_{2}$ - прямые линии, пересекающиеся в одной точке; прямую $l_{0}$ будем считать вертикальной. Пусть $z$ и $z_{1}$ - тангенсы углов наклона прямых $l_{1}$ и $l_{2}$ к горизонтальной прямой. Будем говорить, что $l_{2}$ сопряжсена $l_{1}$ относительно $l_{0}$ (обозначение: $l_{1} \rightarrow l_{2}$ ), если $z$ и $z_{1}$ связаны соотношением

$$
z_{1}=\frac{z^{2}-1}{2 z}
$$

Отметим, что введенное так соотношение сопряжения не реф̆лексивно и не транзитивно.

Полученные результаты о строении спектра гамильтоновой системы с неприводимым интегралом 5-й степени можно сформулировать следуюшим образом. Пусть $l_{0}, l_{1}$ и $l_{2}$ - произвольные три прямые спектра. Тогда либо прямые $l_{1}$ и $l_{2}$ симметричны относительно прямой $l_{0}$, либо выполнено одно из отношений: $l_{1} \rightarrow l_{2}$ или $l_{2} \rightarrow l_{1}$.

В качестве примера рассмотрим случай, когда спектр лежит на пяти прямых, последовательно составляющих друг с другом углы, равные $\pi / 5$. Обозначим их $l_{0}, l_{1}, \ldots, l_{4}$ (рис. 1 ). Покажем, что в этом случае выполнено необходимое условие существования неприводимого интеграла 5 -й степени. Действительно, таблица отношений сопряженности прямых (относительно одной из них, скажем, $l_{0}$ ) выглядит следуюшим образом:

$$
l_{0} \rightarrow l_{0}, \quad l_{1} \rightarrow l_{2}, \quad l_{2} \rightarrow l_{4}, \quad l_{3} \rightarrow l_{1}, \quad l_{4} \rightarrow l_{3} .
$$




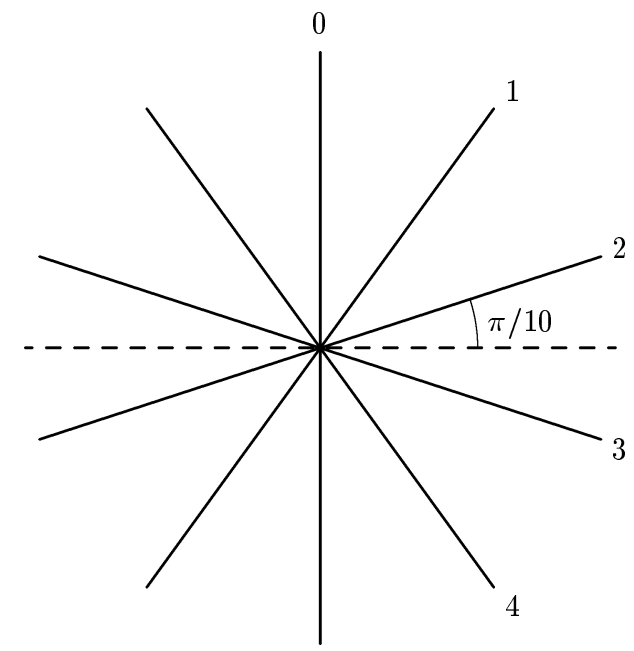

Рис. 1

Зафиксируем прямую $l_{0}$. Тогда две других прямых спектра можно выбрать одним из шести способов:

$$
l_{1} \text { и } l_{4}, \quad l_{2} \text { и } l_{3}, \quad l_{1} \text { и } l_{2}, \quad l_{1} \text { и } l_{3}, \quad l_{2} \text { и } l_{4}, \quad l_{3} \text { и } l_{4} .
$$

В первых двух случаях выбранные прямые симметричны относительно прямой $l_{0}$, а в оставшихся четырех случаях одна из пары прямых сопряжена другой прямой.

Обсудим теперь задачу об интеграле шестой степени. Он имеет вид $F_{6}+F_{4}+$ $F_{2}+F_{0}$, где

$$
F_{6}=a_{0} y_{1}^{6}+a_{2} y_{1}^{4} y_{2}^{2}+a_{4} y_{1}^{2} y_{2}^{4}+a_{5} y_{1} y_{2}^{5}+a_{6} y_{2}^{6} .
$$

Мы предполагаем, что одна из прямых спектра вертикальна. Поэтому в выражении (6.6) коэффициенты $a_{1}$ и $a_{3}$ отсутствуют.

Снова можно предположить, что кроме вертикальной прямой имеются еше две различные прямые спектра, которые однозначно определяются тангенсами $z$ и $z_{1}$ углов наклона этих прямых к горизонтали. Согласно (2.4) и (2.5) вешественное $z$ удовлетворяет двум уравнениям:

$6 a_{0} z^{5}+\left(-2 z^{5}+4 z^{3}\right) a_{2}+\left(-4 z^{3}+2 z\right) a_{4}+\left(5 z^{2}-1\right) a_{5}-6 a_{6} z=0, \quad(6.7)$
$10 a_{0} z^{3}+\left(2 z^{5}-6 z^{3}+2 z\right) a_{2}+\left(-2 z^{5}+6 z^{3}-2 z\right) a_{4}+\left(5 z^{4}-5 z^{2}\right) a_{5}-10 a_{6} z^{3}=0$.

Удобно ввести новые переменные

$$
\gamma_{1}=6 a_{0}-2 a_{2}, \quad \gamma_{2}=4 a_{2}-4 a_{4}, \quad \gamma_{3}=2 a_{4}-6 a_{6} .
$$

В этих обозначениях уравнения (6.7) и (6.8) слегка упрощаются:

$$
\begin{gathered}
\gamma_{1} z^{5}+\gamma_{2} z^{3}+\gamma_{3} z+a_{5}\left(5 z^{2}-1\right)=0 \\
10 \gamma_{1} z^{3}+\gamma_{2}\left(3 z^{5}-4 z^{3}+3 z\right)+10 \gamma_{3} z^{3}+a_{5}\left(30 z^{4}-30 z^{2}\right)=0 .
\end{gathered}
$$


Точно таким же уравнениям, очевидно, удовлетворяет и число $z_{1}$. В итоге мы имеем линейную однородную систему для $\gamma_{1}, \gamma_{2}, \gamma_{3}$ и $a_{5}$. Если определитель этой системы отличен от нуля, то эти переменные равны нулю. Поскольку $a_{1}=a_{3}=0$ и выполнены (6.9), то в этом случае суммы Биркгофа

$$
a_{1}-a_{3}+a_{5} \text { и } a_{0}-a_{2}+a_{4}-a_{6}
$$

одновременно равны нулю. Однако это означает, что многочлен $F_{6}$ делится нацело на многочлен $\left(y_{1}^{2}+y_{2}^{2}\right) / 2$ (кинетическая энергия системы).

Указанный определитель равен

$$
z z_{1}\left(z z_{1}+1\right) \Delta
$$

где $\Delta$ определяется (6.3). Таким образом, возможные положения двух других прямых спектра находятся из условия, что произведение (6.10) равно нулю. Условие $\Delta=0$ уже было проанализировано выше в связи с задачей об интеграле пятой степени. $\mathrm{K}$ этим условиям добавляются два новых соотношения: $z z_{1}=0$ и $z z_{1}+1=0$. Первое из них показывает, что одна из прямых спектра может быть горизонтальной, а второе соотношение является условием ортогональности двух прямых спектра.

Итак, пусть $l_{0}, l_{1}$ и $l_{2}$ - любые три прямые спектра гамильтоновой системы. Если имеется неприводимый интеграл шестой степени, то выполнено одно из следующих условий:

(a) $l_{1}$ или $l_{2}$ ортогональны $l_{0}$,

(b) $l_{1}$ и $l_{2}$ ортогональны,

(c) $l_{1}$ и $l_{2}$ симметричны относительно $l_{0}$,

(d) $l_{1} \rightarrow l_{2}$ или $l_{2} \rightarrow l_{1}$ (относительно $l_{0}$ ).

Рассмотрим пример: шесть прямых спектра отстоят друг от друга на угол $\pi / 6$. Зафиксируем одну из прямых $l_{0}$. Тогда две другие прямые $l_{1}$ и $l_{2}$ можно выделить десятью различными способами. Нетрудно проверить, что для каждого из этих способов вьполнено одно из условий (a)-(d).

\section{§7. Замечания об интегралах произвольной степени}

К задаче о полиномиальных по импульсам интегралах можно подойти с другой стороны. Предположим, что спектр потенциальной энергии лежит на $n$ различных прямых, проходящих через начало координат. Как было отмечено в $\S 2$, в этом случае рассматриваемая динамическая система не допускает нетривиальных дополнительных интегралов степени $k<n$. Таким образом, минимальная возможная степень дополнительного полиномиального интеграла равна $n$.

С другой стороны, как показано в $\S 4$, если система допускает интеграл третьей степени и имеются три различных прямых спектра, то углы между этими прямыми равны $\pi / 3$. Аналогичное замечание справедливо и для интеграла четвертой степени: четыре возможные прямые спектра отстоят друг от друга на угол $\pi / 4$. Оказывается, эти наблюдения можно обобщить. 
ТЕОРема 3. Если имеется полиномиальный интеграл степени $n$, независимый от интеграла әнергии, то п прямых спектра потенииальной әнергии образуют межсду собой угль

$$
\frac{\pi}{n}, \quad \frac{2 \pi}{n}, \quad \ldots, \quad \frac{(n-1) \pi}{n}
$$

Это утверждение указьвает на интересную связь между непрерывной группой симметрий (задаваемой дополнительным интегралом) и дискретньми симметриями динамической системы.

Теорема 3 имеет интересное применение для систем со “стандартной” метрикой (1.2).

СЛЕДСТВИЕ. Предположим, что спектр гамильтоновой системы с кинетической энергией (1.2) лехст на $n \neq 4$ различных прямых, проходящих через начало координат. Тогда эта система не имеет дополнительного полиномиального интеграла степени $n$.

Действительно, как хорошо известно, числа $\operatorname{tg}(\pi / n)$ иррациональны при $n \geqslant 3$ и $n \neq 4$. Трудности рассмотрения особого случая $n=4$ показаны в $\S 5$.

ДокАЗАТЕЛЬСТво ТЕОРЕмЫ 3. Пусть

$$
F_{n}=a_{0} y_{1}^{n}+a_{1} y_{1}^{n-1} y_{2}+\cdots+a_{n} y_{2}^{n}
$$

- старшая однородная форма полиномиального интеграла $n$-й степени. Пусть точка с координатами $u, v$ есть точка спектра. Всегда можно считать, что $v \neq 0$ (после поворота плоскости вокруг начала координат). Отношение $z=u / v$ однозначно определяет прямую спектра, содержащую точку $(u, v)$. По предположению мы имеем $n$ различных таких чисел $z_{1}, \ldots, z_{n}$. Согласно $(2.4)$ и (2.5) эти числа одновременно являются корнями двух многочленов $n$-й степени:

$$
\begin{aligned}
0= & \sum_{i=0}^{n} z^{i}\left((-1)^{i} C_{i+1}^{1} a_{i+1}+(-1)^{i-1} C_{n-i+1}^{1} a_{i-1}\right), \\
0= & \sum_{i=0}^{n} z^{i}\left((-1)^{i} C_{i+3}^{3} a_{i+3}+(-1)^{i-1} C_{n-i-1}^{1} C_{i+1}^{2} a_{i+1}\right. \\
& \left.\quad+(-1)^{i-2} C_{n-i+1}^{2} C_{i-1}^{1} a_{i-1}+(-1)^{i-3} C_{n-i+3}^{3} a_{i-3}\right) .
\end{aligned}
$$

Следовательно, их коэффициенты пропорциональны друг другу. Пусть $\omega-$ отношение коэффициентов при одинаковых степенях $z$ многочленов (7.1) и (7.2).

Пусть $n=2 m$ четно. Случай нечетного $n$ рассматривается аналогично (он даже более простой). Условия пропорциональности коэффициентов разбиваются на две группы замкнутых линейных уравнений:

$$
\begin{array}{r}
\omega\left(-C_{2 j+2}^{1} a_{2 j+2}+C_{n-2 j}^{1} a_{2 j}\right)=-C_{2 j+4}^{3} a_{2 j+4}+C_{n-2 j-2}^{1} C_{2 j+2}^{2} a_{2 j+2} \\
-C_{n-2 j}^{2} C_{2 j}^{1} a_{2 j}+C_{n-2 j+2}^{3} a_{2 j-2},
\end{array}
$$




$$
\begin{aligned}
& \text { где } j=0, \ldots, m-1, \text { и } \\
& \begin{array}{r}
\omega\left(C_{2 j+1}^{1} a_{2 j+1}-C_{n-2 j+1}^{1} a_{2 j-1}\right)=C_{2 j+3}^{3} a_{2 j+3}-C_{n-2 j-1}^{1} C_{2 j+1}^{2} a_{2 j+1} \\
+C_{n-2 j+1}^{2} C_{2 j-1}^{1} a_{2 j-1}-C_{n-2 j+3}^{3} a_{2 j-3},
\end{array}
\end{aligned}
$$

где $j=0, \ldots, m$.

Умножая каждое из уравнений $(7.3)$ на $(-1)^{j}$ и складывая их, приходим к соотношению

$$
\left(\omega C_{n}^{1}+C_{n}^{3}\right)\left[a_{0}-a_{2}+a_{4}-\cdots+(-1)^{m} a_{2 m}\right]=0 .
$$

Аналогично, из уравнений (7.4) выводится равенство

$$
\left(\omega C_{n}^{1}+C_{n}^{3}\right)\left[a_{1}-a_{3}+a_{5}-\cdots+(-1)^{m-1} a_{2 m-1}\right]=0 .
$$

Выражения в квадратных скобках (7.5) и (7.6) - это суммы Биркгофа для многочлена $F_{n}$. Поскольку (по предположению) $F_{n}$ не делится на $\left(y_{1}^{2}+y_{2}^{2}\right) / 2$, то хотя бы одна из этих сумм отлична от нуля. Следовательно,

$$
\omega=-C_{n}^{3} / C_{n}^{1}
$$

Подставляя это значение в (7.4) при $j=0$, получим

$$
a_{1}=\alpha C_{n}^{1}, \quad a_{3}=-\alpha C_{n}^{3},
$$

где $\alpha$ - некоторая ненулевая вещественная постоянная. Из соотношений (7.4) с учетом (7.7) последовательно находим коэффициенты $a$ с нечетными индексами:

$$
a_{2 j+1}=(-1)^{j} \alpha C_{n}^{2 j+1}, \quad 0 \leqslant j \leqslant m-1 .
$$

Отсюда получаем следуюшее соотношение:

$$
C_{2 j+1}^{1} a_{2 j+1}-C_{n-2 j+1}^{1} a_{2 j-1}=(-1)^{j} \alpha n C_{n}^{2 j} .
$$

Соотношение (7.3) при $j=0$ с учетом (7.7) дает

$$
-C_{2}^{1} a_{2}+C_{n}^{1} a_{0}=\beta C_{n}^{1}
$$

где $\beta$ - вещественное число, отличное от нуля.

Из (7.3) и (7.7) получаем последовательно

$$
-C_{2 j+2}^{1} a_{2 j+2}+C_{n-2 j}^{1} a_{2 j}=(-1)^{j} \beta C_{n}^{2 j+1} .
$$

Соотношения (7.8) и (7.9) позволяют преобразовать алгебраическое уравнение (7.1) к следуюшему виду:

$$
n \alpha \sum_{j=0}^{m}(-1)^{j} C_{n}^{2 j} z^{2 j}+\beta \sum_{j=0}^{m-1}(-1)^{j} C_{n}^{2 j+1} z^{2 j+1}=0 .
$$

Учитьвая, что $z=\operatorname{tg} \varphi$, где $\varphi$ - угол наклона прямой спектра к горизонтали, из (7.10) получаем уравнение

$$
n \alpha \cos n \varphi-\beta \sin n \varphi=0 .
$$

Поскольку $\alpha$ и $\beta$ отличны от нуля, то его решениями являются $n$ углов из теоремы 3. Что и требовалось доказать. 


\section{Список литературы}

1. Биркгоф Дж. Д. Динамические системы. М.-Л.: Гостехиздат, 1941.

2. Kozlov $V$. $V$. Integrable and non-integrable Hamiltonian systems // Soviet Sci. Rev. Sect. C Math. Phys. Rev. 1989. V. 8. № 1. P. 1-81.

3. Денисов степенями свободы и торическим конфигурационньм пространством // Матем. заметки. 1998. Т. 64. № 1. С. 37-44.

4. Колокольцов $B . H$. Геодезические потоки на двумерных многообразиях с дополнительным полиномиальным по скоростям первым интегралом // Изв. АН СССР. Сер. матем. 1982. Т. 46. № 5. С. 994-1010.

5. Бабенко И.К., Нехорошев Н.H. О комплексных структурах на двумерных торах, допускающих метрики с нетривиальньм квадратичным интегралом // Матем. заметки. 1995. Т. 58. № 5. С. 643-652.

6. Козлов В. В., Денисова Н. В. Полиномиальные интегралы геодезических потоков на двумерном торе // Матем. сб. 1994. Т. 185. №12. С. 49-64.

7. Козлов В. В., Трещёв Д. В. Об интегрируемости гамильтоновых систем с торическим пространством положений // Матем. сб. 1988. Т. 135 (177). № 1. С. 119-138.

8. Бялый $М$. Л. О полиномиальных по импульсам первых интегралах для механической системы на двумерном торе // Функц. анализ и его прилож. 1987. Т. 21. № 4. С. 64-65.

9. Переломов А. М. Интегрируемые системы классической механики и алгебры Ли. М.: Наука, 1990.

10. Болсинов A. В., Козлов В.В., Фоменко А. Т. Принцип Мопертюи и геодезические потоки на сфере, возникающие из интегрируемых случаев динамики твердого тела // УМН. 1995. Т. 50. №3. С. 3-32.

11. Козлов В. В. Симметрии, топология и резонансы в гамильтоновой механике. Ижевск: Изд-во УдГУ, 1995.

12. Пидкуйко С.И., Стёпин А. М. Полиномиальные интегралы гамильтоновых систем // Докл. АН СССР. 1978. Т. 239. № 1. С. 50-53.

13. Козлов В. В. О полиномиалњыт интегралах системы взаимодействующих частиц // Докл. АН СССР. 1988. Т. 301. № 4. С. 785-788.

14. Пуанкаре A. Новые методы небесной механики // Избранные труды. Т. 1. М.: Наука, 1971.

Московский государственнњй

Поступила в редакцию

университет им. М.В. Ломоносова

21.06.1999 